\title{
Web-based tools for microRNAs involved in human cancer (Review)
}

\author{
FERMÍN MAR-AGUILAR ${ }^{1}$, CRISTINA RODRÍGUEZ-PADILLA ${ }^{2}$ and DIANA RESÉNDEZ-PÉREZ ${ }^{1,2}$ \\ ${ }^{1}$ Departamento de Biología Celular y Genética; ${ }^{2}$ Departamento de Inmunología y Virología, Facultad de Ciencias Biológicas, \\ Universidad Autónoma de Nuevo León, San Nicolás de los Garza, Nuevo León 66451, México
}

Received January 14, 2015; Accepted March 10, 2016

DOI: $10.3892 / \mathrm{ol} .2016 .4446$

\begin{abstract}
MicroRNAs (miRNAs/miRs) are a family of small, endogenous and evolutionarily-conserved non-coding RNAs that are involved in the regulation of several cellular and functional processes. miRNAs can act as oncogenes or tumor suppressors in all types of cancer, and could be used as prognostic and diagnostic biomarkers. Databases and computational algorithms are behind the majority of the research performed on miRNAs. These tools assemble and curate the relevant information on miRNAs and present it in a user-friendly manner. The current review presents 14 online databases that address every aspect of miRNA cancer research. Certain databases focus on miRNAs and a particular type of cancer, while others analyze the behavior of miRNAs in different malignancies at the same time. Additional databases allow researchers to search for mutations in miRNAs or their targets, and to review the naming history of a particular miRNA. All these databases are open-access, and are a valuable tool for those researchers working with these molecules, particularly those who lack access to an advanced computational infrastructure.
\end{abstract}

\section{Contents}

1. Introduction

2. SomamiR DB 2.0

3. miRandola

4. PROGmiR

5. AutomiRDB

6. OncomiRdbB

7. miRCancer

8. CancerNet

9. canEvolve

Correspondence to: Dr Diana Reséndez-Pérez, Departamento de Inmunología y Virología, Facultad de Ciencias Biológicas, Universidad Autónoma de Nuevo León, San Nicolás de los Garza, University Avenue S/N, Nuevo León 66451, México

E-mail: diana.resendezpr@uanl.edu.mx

Key words: microRNAs, cancer, web-based tools, databases, oncomirs

\author{
10. HNOCDB \\ 11. miREC \\ 12. Renal Cancer Gene Database \\ 13. Pancreatic Cancer Database \\ 14. Sarcoma microRNA Expression Database \\ 15. miRBase Tracker \\ 16. Conclusions
}

\section{Introduction}

Since the finding that small non-coding RNAs in Caenorhabditis elegans repress gene expression by binding to mRNA after transcription, our view on gene regulation has changed. These important small regulatory RNAs are known as microRNAs (miRNAs/miRs) $(1,2)$.

miRNA-mediated gene regulation involves 3 steps: i) Processing the transcripts into 22 -nucleotide long RNAs; ii) reaching the target mRNA guided by argonaute (Ago) proteins; and iii) repressing expression by inhibiting or degrading the target mRNA. In the animal kingdom, each species has a number of miRNA genes (3) that control gene expression in the majority of developmental and physiological processes (4).

Among the processes with miRNA-regulated gene expression are the immune response, signal transduction, and cell proliferation and differentiation, as well as other metabolic processes, including fat metabolism and insulin secretion (5). miRNAs mostly affect gene expression by degrading or repressing the target $\mathrm{mRNA}$, although there is evidence that miRNAs can also act as enhancers of gene expression (6). The level of complementarity between mRNA and miRNA determines whether the target gene will be degraded or inhibited; near-perfect complementarity means that the mRNA will be degraded, while imperfect pairing and bulges mean that the mRNA will be inhibited (7). The pathways that miRNAs use to degrade mRNAs are well understood, as they behave similarly to small interfering RNAs (8).

Given their significant role in normal developmental and physiological processes, it follows that miRNAs also play an important role in cancer, either as oncogenes (oncomirs) or tumor suppressor genes. The deregulated expression of miRNA in human cancers has been observed since 2002, first in hematopoietic cancer (9) and later in solid tumors such as 
carcinomas, and colonic and rectal adenomas (10), as well as in malignant brain tumors (11), thyroid (12), breast (13) and lung $(14,15)$ cancer, and hepatocellular carcinomas (16). miRNA expression profiles can be more informative and accurate for identifying certain types of cancer than the currently used methods (17), and miRNA expression profiles can aid in cancer classification and prognosis.

The growing body of transcriptomic data on miRNA expression has fueled the growth of resources that analyze miRNA expression patterns in normal tissues. Certain databases have been created using computational predictions and experimental results, for example miRBase (18), miRNAMap2.0 (19), miRGen (20), miRGator v2.0 (21) and miRecords (22). Other databases, such as TargetScan (2), PicTar (23) and TargetMiner (24), are based on algorithms designed to predict microRNA targets according to the complementary pairing with the target. Another database, TarBase (25), uses validated microRNA targets, while RNAhybrid (26) is based on hybridization between miRNA and mRNA. There are also databases that provide information on various diseases, such as MicroCosm (27), PhenomiR 2.0 (28) and mir2Disease (29). However, few databases are devoted to cancer-related miRNAs. The current review presents several recently published web-based tools for studying the association between miRNA and cancer. These databases are summarized in Table I.

\section{SomamiR DB 2.0}

Circular RNAs (circRNAs) and long non-coding RNA (lncRNA) can occasionally share target mRNAs with miRNAs. When this occurs, these molecules can form a competing endogenous RNA (ceRNA) network. There appears to be an association between the cRNA network and cancer. As miRNA recognition depends on the sequence, it follows that mutations in either miRNAs or targets will affect the miRNA-ceRNA union (30).

SomamiR DB 2.0 (http://compbio.uthsc.edu/SomamiR/) was created to improve on the search of somatic mutations in miRNAs and ceRNAs (lncRNAs, circRNAs and mRNAs). The database searches for somatic mutations associated with cancer and allows researchers to perform a functional search of these mutations.

The database can be searched for somatic mutations in miRNA sequences, and offers the option of using miR2GO. miR2GO can perform a functional analysis of the importance of these mutations in miRNAs. Additionally, miR2GO offers miRmut2GO, an option that checks whether mutations on the miRNA produce changes in the target, and miRpair2GO, which offers a gene ontology analysis for the target gene sets of different miRNAs. SomamiR 2.0 can also search for miRNA mutations that have been experimentally identified by the crosslinking, ligation and sequencing of hybrids. The database can also search for mutations that have been experimentally detected using photoactivatable-ribonucleoside-enhanced crosslinking and immunoprecipitation, and high-throughput sequencing of RNA isolated by crosslinking immunoprecipitation.

Researchers can also search SomamiR 2.0 for predicted mutations on the 3'-untranslated regions (3'UTRs) of target genes, as well as lncRNAs and circRNAs that create or destroy putative target sites on miRNAs. The search for biological pathways affected by mutations in miRNA target sites shows cancer genes associated with Kyoto Encyclopedia of Genes and Genomes pathways. Finally, the database can be searched for mutations on miRNAs associated with cancer, or their target sites.

SomamiR 2.0 can be used to search for somatic and germ-line mutations in miRNAs or targets that are closely related to cancer if the genes were associated with cancer risk using GWAS (Genome-wide association study) o CGAS (candidate-gene association studies) and if there is experimental evidence that the mutation alters the miRNA functions. The mutations that comply with these criteria can be accessed by clicking on experimental evidence linking miRNA related polymorphisms with cancer or experimental evidence linking polymorphisms in miRNA with cancer, respectively. SomamiR is a tool that facilitates the search for cancer-related mutations and can be very a valuable tool in the understanding of this disease $(31,32)$.

\section{3. miRandola}

Extracellular vesicles (exosomes, microvesicles and other membranous vesicles) can be found in a number of bodily fluids, including blood, urine and saliva, and are involved in cancer biology due to the role that they play in intercellular communication. Extracellular vesicles contain a variety of molecules, among which are miRNAs, lncRNAs, mRNAs and proteins, and these molecules are associated with the appearance of cancer, from the start of the tumor to angiogenesis, immunologic vigilance, drug resistance, invasion and metastasis (33).

miRandola (http://mirandola.iit.cnr.it) offers information on the miRNAs present in extracellular vesicles. Extracellular miRNAs are classified into four groups: miRNA-Ago2, miRNA-high-density lipoprotein (HDL), miRNA-exosome and miRNA-circulating. In the latter category are all those miRNAs that cannot be classified in the other 3 categories, as the study did not specify it.

To date, miRandola contains information from 271 studies, yielding 2,681 entries for non-coding RNAs (ncRNAs), among which are 174 miRNAs associated with Ago2, 1,618 circulating miRNAs, 868 miRNAs in exosomes, 21 miRNAs in HDL and 9 miRNAs in microvesicles. The database has 2,179 entries for 12 different types of body fluids: 1,096 for plasma, 983 for serum, 28 for blood, 15 for saliva, 6 for amniotic fluid, 7 for breast milk, 5 for cerebrospinal fluid, 6 for calostrum, 5 for peritoneal fluid, 4 for pleural effusions, 6 for seminal fluid and 18 for urine.

The database has fast and advanced search options. In the fast search, users can search by mature miRNA, ncRNA, miRNA family, type or RNA (Ago, circulating, exosome and HDL), disease, malignant cell line and potential biomarkers. The results can be exported as .xls or .csv. In the advanced search, users can select between miRNA families or mature miRNAs and sample type. The results for this database include links to the miRò database (34), which offers information on the associations between the miRNA and diseases, function, processes and tissues. 


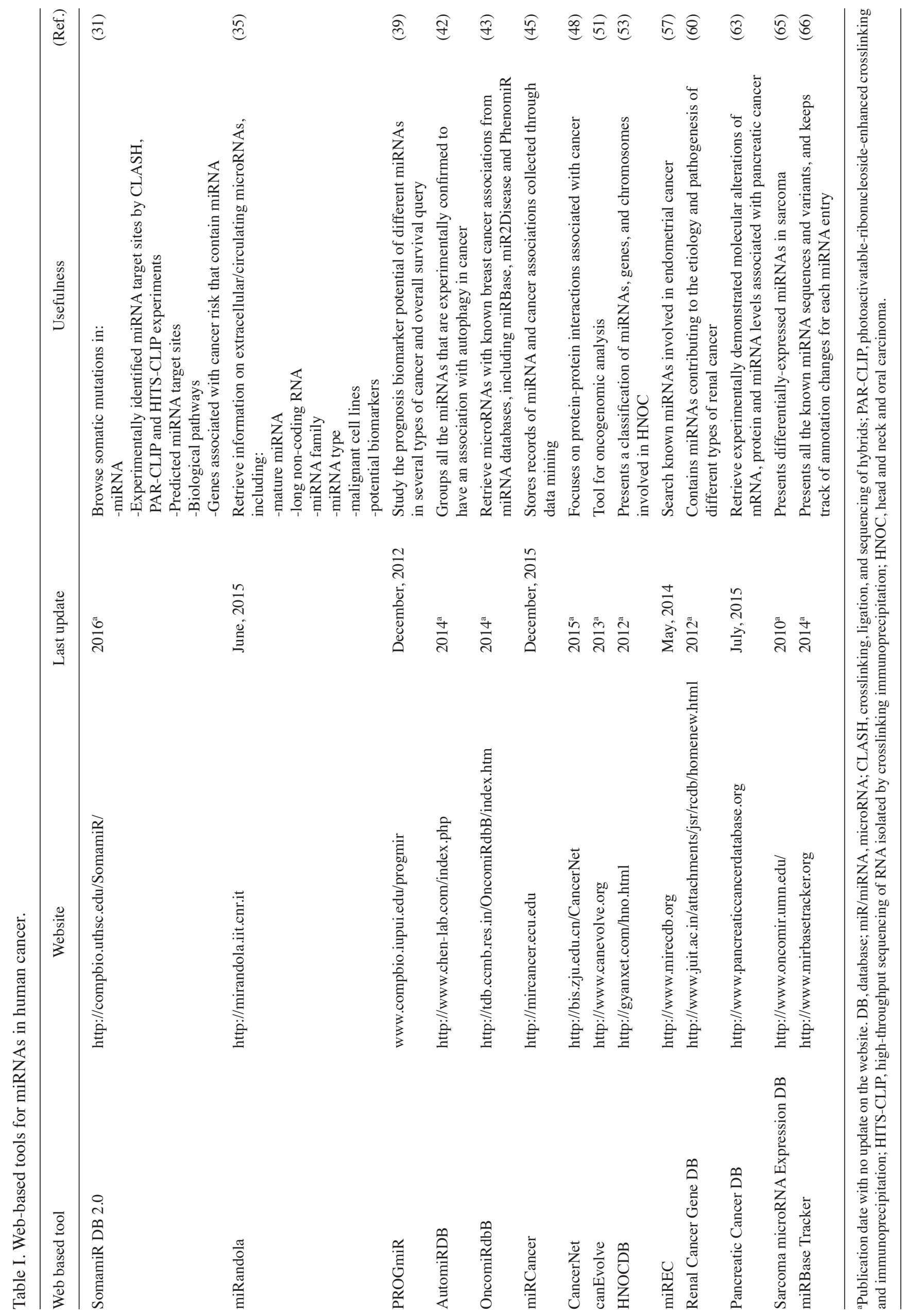


miRandola has two notable tools: miRNAexpress, which makes a systematic comparison of the expression profiles of cellular and extracellular miRNAs, and miMETA, a tool for the meta-analysis of the miRandola database. miRandola specializes in circulating miRNAs, which are increasingly important as non-invasive biomarkers. The database is curated manually, and the data is recovered from the full paper, not just the abstract; this allows miRandola to offer more detailed information, such as method of analysis and a description of the experiment. Finally, the database offers links to other useful databases, including miRBase (www.mirbase.org), miRò (ferrolab.dmi.unict.it/miro/), ExoCarta (www.exocarta.org), and PubMed (www.ncbi.nlm.nih.gov/pubmed) $(35,36)$.

\section{PROGmiR}

PROGmiR (www.compbio.iupui.edu/progmir) allows researchers to study the prognosis biomarker potential of various miRNAs in several types of cancer and overall survival (37). Information from Gene Expression Omnibus and The Cancer Genome Atlas is used, together with information on 16 different types of cancer to create survival Kaplan-Meier plots (38). The tool, which is intended for hypothesis-building only, can offer researchers information regarding the role that a certain miRNA plays in a certain cancer, whether it acts as an oncogene or a tumor suppressor, or both. PROGmiR is freely available online for non-commercial use. The database can be searched by miRNA ID, and then the users can select the cancer type for which they want to see the prognosis, or the 3- or 5-year survival rate. Each plot is created from one database, so as to avoid bias or other issues. Currently, the database has the profiles of 1,050 miRNAs from 3,117 samples from 16 types of cancer (39).

\section{AutomiRDB}

Autophagy is a cellular process that can lead to cell death, where a cell destroys proteins and other substances present in its cytoplasm. Autophagy can prevent cells from becoming cancerous, but it can also protect cancer cells from anticancer drugs (40). There is evidence that miRNAs have a significant role in autophagy regulation pathways, and this growing research field can aid in the development of novel therapies against cancer (41).

AutomiRDB (http://www.chen-lab.com/index.php) groups all the miRNAs that are experimentally confirmed to have an association with autophagy in cancer (42). The information in the database allows users to associate human miRNAs with target genes or proteins in different malignancies. To develop AutomiRDB, the published literature was searched for genes involved in autophagy in cancer, and predictive analysis was performed to determine which miRNAs would target those genes. The database includes 493 miRNAs associated with 90 genes or proteins associated with autophagy in 18 different types of cancer. The database is simple to use; the user only has to type the name of the miRNA in the query. The results page contains hyperlinks that lead to more information in other databases, including OMIM (http://www.omim.org) and UNIPROT (http://www.uniprot.org), among others (42).

\section{OncomiRdbB}

OncomiRdbB (tdb.ccmb.res.in/OncomiRdbB/index.htm) (43) was built from 782 human and 246 mouse microRNAs that possessed known associations with breast cancer, which were retrieved from miRNA databases, including miRBase (18), miR2Disease (29) and PhenomiR 2.0 (28). The findings were validated using Taqman low density arrays that consisted of 667 human microRNAs and LNA ${ }^{\mathrm{TM}}$ arrays using human breast cancer samples. These tests yielded $\sim 400$ breast cancer miRNAs, as well as 34 miRNA sequences that had not been classified as such. To find the targets of the miRNAs involved in breast cancer, the developers compared the 3'UTR, 5'UTR and exonic regions of genes from different pathways using miRanda. This analysis revealed 711 potential target genes for humans and 490 for mice, which is more than can be found in other databases.

OncomiRdbB is a freely available web interphase. Users can search miRNAs by name, accession number, target gene or sequence. The genes are classified according to their signaling pathway, and the database includes information on gene location within the chromosome, and alignment between the miRNA and the target, as well as links to the parental databases where the genes were retrieved (43).

\section{7. miRCancer}

miRCancer (http://mircancer.ecu.edu) is a database that stores records of miRNA and cancer associations collected through data mining (44). A rule-based approach was devised to analyze the title and abstract of 26,414 publications and to find full sentences or phrases that included the names of the miRNA and the cancer type, and any expression terms. The results of this data mining process were then corroborated by hand. miRCancer has records of $>3,764$ miRNA-cancer associations from 2,611 publications, which amounts to 236 miRNA expression profiles from 176 human cancers.

The rules used in text mining for this database resulted in a recall rate of $78 \%$. One of the reasons for certain studies not being recalled can be attributed to the manner in which the study was written. The method used requires that the miRNA-cancer association be stated clearly, preferably in one sentence.

miRCancer is freely accessible online, and the database can be searched by miRNA name or cancer type, or a combination of both (45).

\section{CancerNet}

The biological processes that keep a cell in homeostasis result from a number of interactions between proteins. The alteration of these interactions can lead to pathological processes such as cancer (46).

There are several databases that deal with protein interactions, but CancerNet (http://bis.zju.edu.cn/CancerNet) is the first database to focus on the protein-protein interactions associated with cancer. As miRNAs have an important role in the mechanisms that regulate protein expression, building a database than integrates the miRNA regulation network with regard to specific cancer types is an important contribution. 
miRNAs can act in synergy, i.e., several miRNAs can act on a single molecule, and this synergy can be different depending on the type of cancer (47). CancerNet takes this synergy into consideration and provides molecular interactions for 33 cancer types. The synergistic interactions included are 185,589 protein-protein interactions, 3,249,385 miRNA-target gene interactions and miRNA-miRNA interactions.

The CancerNet platform is simple to use and the queries can be made by molecule name and type of cancer, or by entering two molecules to find whether they have synergistic interactions. The results show a list with detailed information on these interactions, expression levels, functional similarity scores and the specificity of each interaction. The results can be viewed as a graph. Another function, the 'GO Enrichment Analysis' allows users to investigate the biological function of the genes that are of interest to them.

Applications for CancerNet include the study of specific interactions in cancer, and the study of the interactions between poorly annotated miRNAs and protein-coding genes (48).

\section{9. canEvolve}

Researchers working with cancer have benefited from the advances in the field of next-generation sequencing technology. There has been a massive amount of information derived from the genomic studies of healthy people and patients with certain diseases. Researchers searching for differences in the expression of miRNAs or genes in different types of cancer can occasionally struggle to find answers in the huge amount of data yielded by these technologies $(49,50)$.

CanEvolve (http://www.canevolve.org) (51) is a database that can aid cancer researchers in answering their questions in a user-friendly manner by assisting them in searching through the data generated by massive sequencing experiments. The current version of canEvolve holds data from $>18,000$ patients in 127 datasets. The datasets include information such as gene expression, copy number alterations, miRNA expression, mutation, protein expression and protein-protein interactions. The database also has information on the differential expression of genes and miRNAs, changes in the number of copies, co-expression data, protein-protein interactions, metabolic and signaling pathways, and targets for transcription factors and miRNAs. The database also uses $>200$ algorithms, including LIMMA, dsChipSNP and ARACNE, to analyze different types of cancer.

CanEvolve can be searched by selecting the type of analysis and then the type of cancer and study. Next, users can enter genes or selected pathways, and click on 'get results'. Results are shown as a heat map, graph or network depending on the desired analysis. The results can be downloaded as tables or R-data objects.

This database allows users to perform different types of integrative analysis and identify genes that are believed to induce or regulate tumorigenesis. It is also possible to perform a pathway meta-analysis from multiple studies of differentially-expressed genes and users are allowed to choose to review the enrichment of gene sets derived from MsigDB. CanEvolve is an excellent tool for oncogenomic analysis and its tools will be extremely useful in the analysis of the huge amount of data that is being generated (51).

\section{HNOCDB}

Head and neck and oral carcinomas (HNOCs) include oral, tongue, salivary gland, thyroid, pharyngeal, hypopharyngeal, nasopharyngeal, oropharyngeal and laryngeal cancer. These cancers are the sixth most common cancer in the world and are difficult to treat due to their location (52). Therefore, great efforts are being made to improve treatment and patient overlife.

HNOCDB (http://gyanxet.com/hno.html) (53) was created with information on genes and miRNAs involved in HNOC that have been validated. The genes were classified according to type of cancer, expression profile, miRNA ID and location in the chromosome. The database includes 451 genes and 109 miRNAs associated with HNOC.

Information can be accessed through 3 main links: 'Genes', 'miRNAs' and 'chromosomes', and then users can choose to see the association of the selected items with a certain type of cancer. In the chromosome section, users can choose the desired chromosome and then choose genes or miRNAs to study. In each section, the results are presented in a table that includes identifying data for the gene or miRNA, function and its alterations in this cancer.

HNOCDB is a database that presents a good classification of the miRNAs, genes and chromosomes involved in HNOC, and is a valuable tool for researchers that study this type of cancer (53).

\section{MiREC}

Endometrial cancer (EC) is one of the most common gynecological cancers in developing countries (54), and as in all the other types of cancer that have been studied, EC has altered miRNA expression $(55,56)$. Furthermore, as with all the other types of cancer, it is important to have a database that integrates all the information on miRNAs, gene annotation and expression, and the biomedical literature associated with this disease.

The miREC database (http://www.mirecdb.org) (57) has gathered information on all the known miRNAs involved in EC either by in silico prediction or experimental validation, which allows us to observe how miRNAs regulate genes in EC. The database (V 2.0) has information on 920 genes and 228 miRNAs. This information includes target genes and miRNAs, references to published scientific studies, literature citations for genes, miRNAs and gene-miRNA associations.

The database can be searched by gene or miRNA name, and has an advanced search option where users can introduce several parameters to refine their search. Results are presented in a table with links to other databases for more information. This database can also offer information on miRNA clusters through the identification of the functional correlation of associated miRNAs. This type of analysis is extremely useful, as it has been observed that miRNAs from the same cluster have common target genes, and that they can even be coexpressed and transcribed like a polycistron. There are databases that can do similar things, but miREC specializes in EC, and is a valuable tool for researchers looking for novel diagnostic tools and treatments for this disease (57). 


\section{Renal Cancer Gene Database}

Renal cell carcinoma (RCC) is a common and often lethal urological cancer complicated by resistance to chemotherapy and radiotherapy (58). Although several molecular markers have been identified for RCC diagnosis and prognosis, it is not possible to predict tumor aggressiveness and metastasis (59).

To study renal cancer data, the Renal Cancer Gene Database integrated 240 protein-coding and 269 miRNAs associated with the pathogenesis of different RCC forms. Genes in this database are classified with regard to alteration in RCC, and deregulated miRNA is also included, with information associated with RCC type and metastatic or prognostic importance with common and unique miRNAs to RCC and other cancers.

The Renal Cancer Gene Database can be freely accessed (www.juit.ac.in/attachments/jsr/rcdb/homenew.html) (60) and uses a keyword search. Users can retrieve a list of genes with specific category and chromosomal location indexed by research studies and complemented with public databases such as Swissprot and Refseq, among others. In addition, users can perform comparative studies to find which genes are shared with other cancers and which genes are unique to RCC. This database is a good resource for researchers and physicians working in renal carcinoma (60).

\section{Pancreatic Cancer Database}

Pancreatic cancer is a leading cause of cancer-associated mortality worldwide (61). A wide range of alterations at the genome, transcriptome and proteome level have been reported for this malignancy. Although expression studies have proposed potential biomarkers for the diagnosis and prognosis of pancreatic cancer (62), there are no repositories and it is difficult to analyze a single gene in the different data and publications.

The Pancreatic Cancer Database provides an integrated web-based resource (www.pancreaticcancerdatabase.org) (63) containing data on the altered expression of 3,481 total genes in pancreatic cancer, from which 703 genes are altered at the mRNA and protein levels, 570 genes are altered at the protein level only and 1,982 genes are altered at the mRNA level only. In addition, the Pancreatic Cancer Database also contains 226 miRNAs associated with pancreatic cancer tissues and cell lines with an external link to miRBase. The Pancreatic Cancer Database was constructed using PHP (http://www.php.net) as a server and MySQL (http://www.mysql.com) as a storage system.

Users can quickly access the Pancreatic Cancer Database with genes, proteins, molecular alterations, type of cancer, different cell lines and experimental methods using the 'browse' option to navigate through the alterations described at the RNA, protein or miRNA levels (63).

\section{Sarcoma microRNA Expression Database}

Human sarcomas are rare, aggressive and invasive tumors that comprise a number of histological subtypes, with few markers for diagnostic and classification. miRNA expression patterns have been proposed as novel biomarkers for diagnosis, classification and prognosis in tumors, but are poorly understood in sarcomas (64).
The Sarcoma microRNA Expression Database (www.oncomir.umn.edu/) (65) was developed as a repository of human sarcoma miRNA expression patterns to address the lagoons in biological and bioinformatics knowledge. This database allows quick access to miRNA expression profiles of 310 tumor tissue samples that represent 22 different types of sarcomas.

The Sarcoma microRNA Expression Database presents the results as a heat map with text and numerical formats, and also provides statistical fold changes and P-values for differentially-expressed miRNAs. Since numerical values can be difficult to interpret, the heat map presents the option of color-coding to represent data in absolute and relative formats (65).

\section{5. miRBase Tracker}

miRBase (www.mirbasetracker.org) (66) is a database that includes all the known miRNA sequences and variants, and keeps track of annotation changes for each miRNA entry. Due to the large amount of research on miRNAs, the number of sequences and annotations has increased, from 218 precursor and 218 mature miRNAs from five different species in 2002 , to 28,645 precursor and 35,828 mature miRNAs from 223 species in 2014.

The rapid growth in the number of miRNA sequences and annotation, as well as the identification of isomiRS resulted in a degree of confusion with miRNA identity. A proportion of this confusion comes from the use of outdated miRNA names in as much as $25 \%$ of the publications, as well as in ambiguities such as using the same name for different miRNA sequences.

The miRBase Tracker works with the miRBase database by keeping track of all the changes undergone by a miRNA sequence through different versions of miRBase in a MySQL base. Users can search the database by miRNA history to observe all the changes and annotations the sequence has gone through. An miRNA update search will yield the most recent annotation for the sequence, and a search by miRBase release compares precursor and mature miRNAs from any species between two miRBase releases, showing the changes undergone by the sequence (66).

\section{Conclusions}

This review shows a variety of user-friendly, open-access web-based tools that connect miRNAs with cancer through experimentally supported results. Although the majority of aspects of the mechanisms surrounding the interactions between miRNAs and cancer remain unknown, these databases can assist researchers to answer these questions. Databases focused on a particular type of cancer such as HNOCDB, miREC, the Renal Cancer Gene Database, the Pancreatic Cancer Database and the Sarcoma microRNA Expression Database are useful to those researchers who want to study the role of miRNAs in cancer biogenesis and evolution. Other databases (CanEvolve, CancerNet, miRCancer, OncomiRdbd, AutomiRDb and ProgmiR) that study the role of miRNAs in different tumors can be used to see the bigger picture of a particular miRNA in different pathologies. 
miRandola and SomamiR are general databases that could play an important role in cancer research. MiRandola presents information on circulating miRNAs that may have an important role in metastasis and may be used to develop non-invasive biomarkers, while SomamiR allows researchers to find mutations that could lead to the development of malignancies.

The miRBase Tracker is not a cancer database, but it was included due to its efforts to unify miRNA naming conventions. This database allows those researchers working with miRNAs to track the name of a particular molecule through the years, thus increasing the scope of the research.

These web-based tools concerning miRNAs and cancer are a central resource for researchers; they are extremely useful in the validation of these targets and in the development of novel clinical cancer biomarkers, and ultimately, novel therapeutic interventions. More importantly, these databases assist researchers in navigating the enormous amounts of data available on miRNAs and cancer by providing curated, specific information in one place.

\section{Acknowledgments}

The authors would like to thank Ms. Alejandra Arreola-Triana for providing an editorial review of the original manuscript, and the Department of Inmmunology and Virology of the Biological Sciences School of the Autonomous University of Nuevo León (Nuevo León, México) for providing financial support.

\section{References}

1. Lai EC: Micro RNAs are complementary to 3'UTR sequence motifs that mediate negative post-transcriptional regulation. Nat Genet 30: 363-364, 2002.

2. Lewis BP, Shih IH, Jones-Rhoades MW, Bartel DP and Burge CB: Prediction of mammalian microRNA targets. Cell 115: 787-798, 2003.

3. Flynt AS andLaiEC: Biological principles of microRNA-mediated regulation: Shared themes amid diversity. Nat Rev Genet 9: $831-842,2008$

4. Ambros V, Lee RC, Lavanway A, Williams PT and Jewell D: MicroRNAs and other tiny endogenous RNAs in C. elegans. Curr Biol 13: 807-818, 2003.

5. Ambros V: MicroRNA pathways in flies and worms: Growth, death, fat, stress and timing. Cell 113: 673-676, 2003

6. Huntzinger E and Izaurralde E: Gene silencing by microRNAs: Contributions of translational repression and mRNA decay. Nat Rev Genet 12: 99-110, 2011.

7. Hutvagner G and Zamore PD: A microRNA in a multiple-turnover RNAi enzyme complex. Science 297: 2056-2060, 2002.

8. Tuschl T, Zamore PD, Lehmann R, Bartel DP and Sharp PA: Targeted mRNA degradation by double-stranded RNA in vitro. Genes Dev 13: 3191-3197, 1999.

9. Calin GA, Dumitru CD, Shimizu M, Bichi R, Zupo S, Noch E, Aldler H, Rattan S, Keating M, Rai K, et al: Frequent deletions and down-regulation of micro- RNA genes miR15 and miR16 at 13q14 in chronic lymphocytic leukemia. Proc Natl Acad Sci USA 99: 15524-15529, 2002.

10. Michael MZ, O'Connor SM, van Holst Pellekaan NG, Young GP and James RJ: Reduced accumulation of specific microRNAs in colorectal neoplasia. Mol Cancer Res 1: 882-891, 2003.

11. Chan JA, Krichevsky AM and Kosik KS: MicroRNA-21 is an antiapoptotic factor in human glioblastoma cells. Cancer Res 65: 6029-6033, 2005

12. Iorio MV, Ferracin M, Liu CG, Veronese A, Spizzo R, Sabbioni S, Magri E, Pedriali M, Fabbri M, Campiglio M, et al: MicroRNA gene expression deregulation in human breast cancer. Cancer Res 65: 7065-7070, 2005 .
13. He H, Jazdzewski K, Li W, Liyanarachchi S, Nagy R, Volinia S, Calin GA, Liu CG, Franssila K, Suster S, et al: The role of microRNA genes in papillary thyroid carcinoma. Proc Natl Acad Sci USA 102: 19075-19080, 2005.

14. Takamizawa J, Konishi H, Yanagisawa K, Tomida S, Osada H, Endoh H, Harano T, Yatabe Y, Nagino M, Nimura Y, et al: Reduced expression of the let-7 microRNAs in human lung cancers in association with shortened postoperative survival. Cancer Res 64: 3753-3756, 2004

15. Hayashita Y, Osada H, Tatematsu Y, Yamada H, Yanagisawa K, Tomida S, Yatabe Y, Kawahara K, Sekido Y and Takahashi T: A polycistronic microRNA cluster, miR-17-92, is overexpressed in human lung cancers and enhances cell proliferation. Cancer Res 65: 9628-9632, 2005 .

16. Murakami Y, Yasuda T, Saigo K, Urashima T, Toyoda H, Okanoue $\mathrm{T}$ and Shimotohno K: Comprehensive analysis of microRNA expression patterns in hepatocellular carcinoma and non-tumorous tissues. Oncogene 25: 2537-2545, 2006.

17. Lu J, Getz G, Miska EA, Alvarez-Saavedra E, Lamb J, Peck D, Sweet-Cordero A, Ebert BL, Mak RH, Ferrando AA, et al: MicroRNA expression profiles classify human cancers. Nature 435: 834-838, 2005.

18. Griffiths-Jones S, Grocock RJ, van Dongen S, Bateman A and Enright AJ: miRBase: microRNA sequences, targets and gene nomenclature. Nucleic Acids Res 34: D140-D144, 2006.

19. Hsu PW, Huang HD, Hsu SD, Lin LZ, Tsou AP, Tseng CP, Stadler PF, Washietl S and Hofacker IL: miRNAMap: Genomic maps of microRNA genes and their target genes in mammalian genomes. Nucleic Acids Res 34: D135-D139, 2006.

20. Alexiou P, Vergoulis T, Gleditzsch M, Prekas G, Dalamagas T, Megraw M, Grosse I, Sellis T and Hatzigeorgiou AG: miRGen 2.0: A database of microRNA genomic information and regulation. Nucleic Acids Res 38: D137-D141, 2010.

21. Nam S, Kim B, Shin S and Lee S: miRGator: An integrated system for functional annotation of microRNAs. Nucleic Acids Res 36: D159-D164, 2008.

22. Xiao F, Zuo Z, Cai G, Kang S, Gao X and Li T: miRecords: An integrated resource for microRNA-target interactions. Nucleic Acids Res 37: D105-D110, 2009.

23. Krek A, Grün D, Poy MN, Wolf R, Rosenberg L, Epstein EJ, MacMenamin P, da Piedade I, Gunsalus KC, Stoffel M and Rajewsky N: Combinatorial microRNA target predictions. Nat Genet 37: 495-500, 2005

24. Bandyopadhyay S and Mitra R: TargetMiner: microRNA target prediction with systematic identification of tissue-specific negative examples. Bioinformatics 25: 2625-2631, 2009.

25. Sethupathy P, Corda B and Hatzigeorgiou AG: TarBase: A comprehensive database of experimentally supported animal microRNA targets. RNA 12: 192-197, 2006.

26. Kruger J and Rehmsmeier M: RNAhybrid: microRNA target prediction easy, fast and flexible. Nucleic Acids Res 34: W451-W454, 2006.

27. Griffiths-Jones S, Saini HK, van Dongen S and Enright AJ: miRBase: Tools for microRNA genomics. Nucleic Acids Res 36: D154-D158, 2008.

28. Ruepp A, Kowarsch A, Schmidl D, Buggenthin F, Brauner B, Dunger I, Fobo G, Frishman G, Montrone C and Theis FJ: PhenomiR: A knowledgebase for microRNA expression in diseases and biological processes. Genome Biol 11: R6, 2010.

29. Jiang Q, Wang Y, Hao Y, Juan L, Teng M, Zhang X, Li M, Wang $\mathrm{G}$ and Liu Y: miR2Disease: A manually curated database for microRNA deregulation in human disease. Nucleic Acids Res 37: D98-D104, 2009.

30. Chen Y, Li C, Tan C and Liu X: Circular RNAs: A new frontier in the study of human diseases. J Med Genet 2016: jmedgenet-2016-103758, 2016 (Epub ahead of print).

31. Bhattacharya A and Cui Y: SomamiR 2.0: A database of cancer somatic mutations altering microRNA-ceRNA interactions. Nucleic Acids Res 44: D1005-D1010, 2016.

32. Bhattacharya A, Ziebarth JD and Cui Y: SomamiR: A database for somatic mutations impacting microRNA function in cancer. Nucleic Acids Res 41: D977-D982, 2013.

33. Kosaka N: Decoding the secret of cancer by means of extracellular vesicles. J Clin Med 5: E22, 2016.

34. Laganà A, Forte $\mathrm{S}$, Giudice $\mathrm{A}$, Arena MR, Puglisi PL, Giugno R, Pulvirenti A, Shasha D and Ferro A: miRò: A miRNA knowledge base. Database (Oxford) 2009: bap008, 2009.

35. Russo F, Di Bella S, Nigita G, Macca V, Laganà A, Giugno R, Pulvirenti A and Ferro A: miRandola: Extracellular circulating microRNAs database. PLoS One 7: e47786, 2012. 
36. Russo F, Di Bella S, Bonnici V, Laganà A, Rainaldi G, Pellegrini M, Pulvirenti A, Giugno R and Ferro A: A knowledge base for the discovery of Russo function, diagnostic potential and drug effects on cellular and extracellular miRNAs. BMC Genomics 15 (Suppl 3): S4, 2014.

37. Tiberio P, Callari M, Angeloni V, Daidone MG and Appierto V: Challenges in using circulating miRNAs as cancer biomarkers. Biomed Res Int 2015: 731479, 2015.

38. Ludbrook J and Royse AG: Analysing clinical studies: Principles, practice and pitfalls of Kaplan-Meier plots. ANZ J Surg 78 204-210, 2008.

39. Goswami CP and Nakshatri H: PROGmiR: A tool for identifying prognostic miRNA biomarkers in multiple cancers using publicly available data. J Clin Bioinforma 2: 23, 2012.

40. Mathew R, Karantza-Wadsworth V and White E: Role of autophagy in cancer. Nat Rev Cancer 7: 961-967, 2007.

41. Jing Z, Han W, Sui X, Xie J and Pan H: Interaction of autophagy with microRNAs and their potential therapeutic implications in human cancers. Cancer Lett 356: 332-338, 2015.

42. Chen Y, Huang J and Liu B: AutomiRDB: A web resource connecting microRNAs and autophagy in cancer. Apoptosis 20 : 1016-1017, 2015.

43. Khurana R, Verma VK, Rawoof A,Tiwari S,NairRA,Mahidhara G, Idris MM, Clarke AR and Kumar LD: OncomiRdbB: A comprehensive database of microRNAs and their targets in breast cancer. BMC Bioinformatics 15: 15, 2014.

44. Frixa T, Donzelli S and Blandino G: Oncogenic microRNAs: Key players in malignant transformation. Cancers (Basel) 7 : 2466-2485, 2015.

45. Xie B,Ding Q,Han H and Wu D: miRCancer: A microRNA-cancer association database constructed by text mining on literature. Bioinformatics 29: 638-644, 2013.

46. Morimoto RI and Cuervo AM: Protein homeostasis and aging: Taking care of proteins from the cradle to the grave. J Geronto A Biol Sci Med Sci 64: 167-170, 2009.

47. Chen B, Duan L, Yin G, Tan J and Jiang X: Simultaneously expressed miR-424 and miR-381 synergistically suppress the proliferation and survival of renal cancer cells - Cdc2 activity is up-regulated by targeting WEE1. Clinics (Sao Paulo) 68: 825-833, 2013.

48. Meng X, Wang J, Yuan C, Li X, Zhou Y, Hofestädt R and Chen M: CancerNet: A database for decoding multilevel molecular interactions across diverse cancer types. Oncogenesis 4: e177, 2015.

49. Kwon SM, Cho H, Choi JH, Jee BA, Jo Y and Woo HG: Perspectives of integrative cancer genomics in next generation sequencing era. Genomics Inform 10: 69-73, 2012.

50. Pimentel F, Bonilla P, Ravishankar YG, Contag A, Gopal N, LaCour S, Lee T and Niemz A: Technology in microRNA profiling: Circulating microRNAs as noninvasive cancer biomarkers in breast cancer. J Lab Autom 20: 574-588, 2015.
51. Samur MK, Yan Z, Wang X, Cao Q, Munshi NC, Li C and Shah PK: canEvolve: A web portal for integrative oncogenomics. PLoS One 8: e56228, 2013.

52. Marur S and Forastiere AA: Head and neck squamous cell carcinoma: Update on epidemiology, diagnosis, and treatment. Mayo Clin Proc 91: 386-396, 2016.

53. Mitra S, Das S, Das S, Ghosal S and Chakrabarti J: HNOCDB: A comprehensive database of genes and miRNAs relevant to head and neck and oral cancer. Oral Oncol 48: 117-119, 2012.

54. Nachajova M, Mersakova S, Sivakova J, Krivus S, Szepe P, Hatok J and Adamkov M: New molecular aspects of endometrial carcinoma. Neuro Endocrinol Lett 36: 638-643, 2015.

55. Sianou A, Galyfos G, Moragianni D, Andromidas P, Kaparos G, Baka S and Kouskouni E: The role of microRNAs in the pathogenesis of endometrial cancer: A systematic review. Arch Gynecol Obstet 292: 271-282, 2015.

56. Li S, Zhang J and Wan X: Role of miRNAs in endometrial cancer. Histol Histopathol 30: 539-548, 2015.

57. Ulfenborg B, Jurcevic S, Lindlof A, Klinga-Levan $\mathrm{K}$ and Olsson B: miREC: A database of miRNAs involved in the development of endometrial cancer. BMC Res Notes 8: 104, 2015.

58. Mourad WF, Dutcher J and Ennis RD: State-of-the-art management of renal cell carcinoma. Am J Clin Oncol 37 498-505, 2014.

59. Arsanious A, Bjarnason GA and Yousef GM: From bench to bedside: Current and future applications of molecular profiling in renal cell carcinoma. Mol Cancer 8: 20, 2009.

60. Ramana J: RCDB: Renal Cancer Gene Database. BMC Res Notes 5: 246, 2012

61. Kourie HR, Gharios J, Elkarak F, Antoun J and Ghosn M: Is metastatic pancreatic cancer an untargetable malignancy? World J Gastrointest Oncol 8: 297-304, 2016.

62. Le N, Sund M and Vinci A; GEMS collaborating group of Pancreas 2000: Prognostic and predictive markers in pancreatic adenocarcinoma. Dig Liver Dis 48: 223-230, 2016.

63. Thomas JK, Kim MS, Balakrishnan L, Nanjappa V, Raju R, Marimuthu A, Radhakrishnan A, Muthusamy B, Khan AA, Sakamuri S, et al: Pancreatic Cancer Database: An integrative resource for pancreatic cancer. Cancer Biol Ther 15: 963-967, 2014.

64. Lim HJ and Yang JL: Regulatory roles and therapeutic potential of microRNA in sarcoma. Crit Rev Oncol Hematol 97: 118-130, 2016.

65. Sarver AL, Phalak R, Thayanithy V and Subramanian S: S-MED: Sarcoma microRNA expression database. Lab Invest 90: 753-761, 2010.

66. Van Peer G, Lefever S, Anckaert J, Beckers A, Rihani A, Van Goethem A, Volders PJ, Zeka F, Ongenaert M, Mestdagh P and Vandesompele J: miRBase Tracker: Keeping track of microRNA annotation changes. Database (Oxford) 2014: bau080, 2014. 